\title{
Not all Rural Areas Are Created Equal: Smoking in Rural America Across Four Categories of an Urban/Rural Continuum
}

\author{
G. Lopez'; H. Mattie 2; E. Culakova'1, C. Kamen', N. Gilmore', M. Janelsins', G. Morrow' \\ ${ }^{1}$ Deparment of Surgery (Cancer Control), University of Rochester Medical Center \\ ${ }^{2}$ Department of Biostatistics, Harvard T.H. Chan School of Public Health
}

ROCHESTER

HARVARD T.H.CHAN

SCHOOL OF PUBLIC HEALTH

\section{Background}

- Tobacco is leading preventable cause of death in U.S.

- Rural populations continue to have higher prevalence of smoking, compared to urban dwellers

- Little is known about smoking disparities across rural categories

\section{Objective}

In this study, we analyze the odds of smoking across four rural-urban categories and the role race/ethnicity plays in this relationship.

\section{Results}

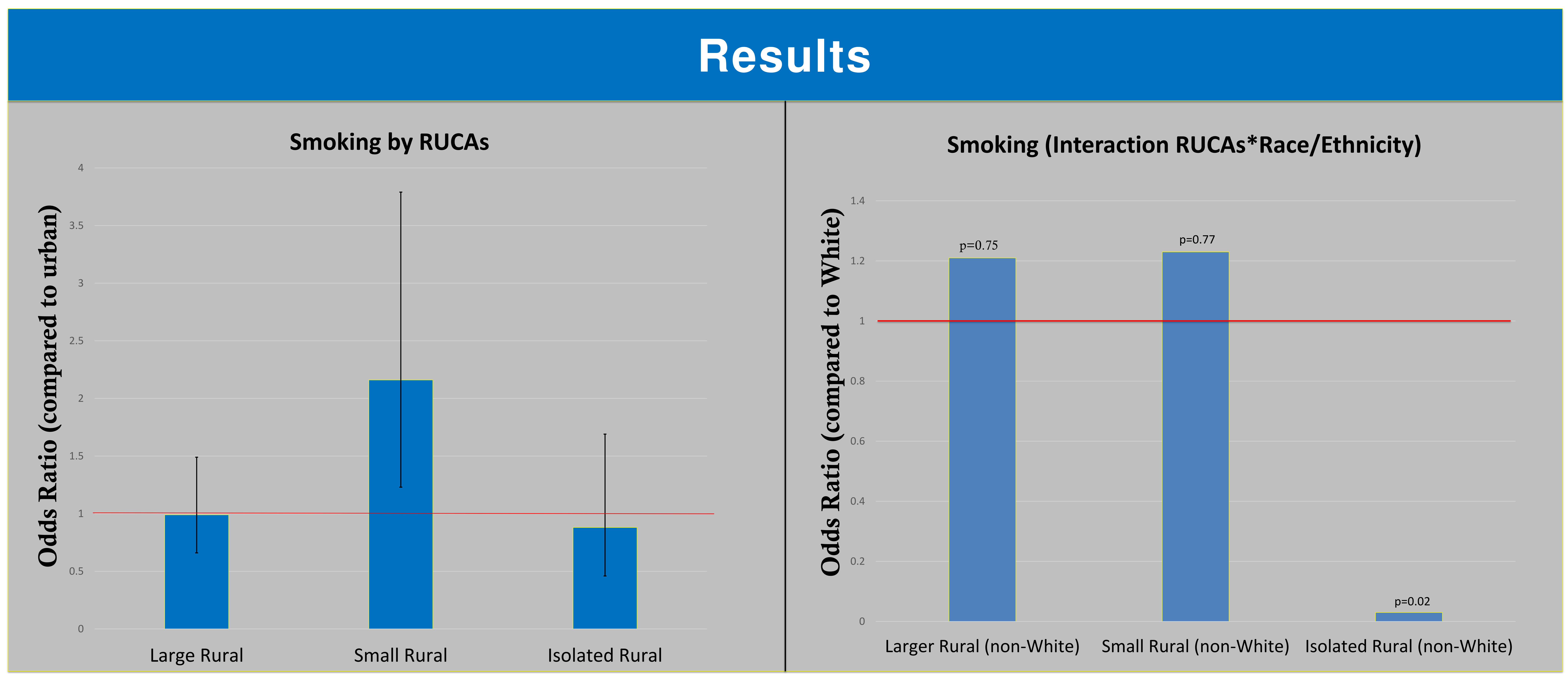

- Using Rural-Urban Commuting Area Codes (RUCAs), from the 2018 Health Information National Trends Survey (HINTS-5) database $(n=5,099)$ we analyze the odds of smoking across four locations: urban, large-rural, small-rural, and isolated-rural areas.

- We test for the interaction of race and rurality to see if the association between RUCAs and smoking differs for Whites and non-Whites.

- Using an established social determinants framework, a series of logistic regression models were fit to estimate odds ratios (see handout).

\section{Conclusions}

- Living in a small rural town is associated with $\mathbf{2 . 1 6}$ times the odds of smoking, compared to urban $(\mathrm{p}<0.01)$

- Non-Whites living in isolated rural areas are at $\mathbf{0 . 0 3}$ times the odds of smoking compared to Whites in isolated rural areas $(\mathrm{p}<0.05)$. 